Audience Psychology:
Measuring Emotional Response of Audience based on Heart Rate
Variability

Part A:

Gender: M / F Age:____ Occupation:

Phone: iPhone / Android (pls specify

Part B:

1. What is your overall rating of the clip (s)

( 1 is the worst while 10 is the best $)=$

2. Which plot/part
a. is the happiest?
b. is the saddest?
c. is the most memorable?
d. is the most boring?

3. Will you recommend this compilation of $\mathrm{CM}$ to the others?

4. Have you watched this clip before?

Part C:

1. What is your overall rating of the clip (s)

( 1 is the worst while 10 is the best $)=$

2. Which plot/part is
a. the happiest?
b. Is the saddest?
c. is the most memorable?
d. is the most boring?

3. Will you recommend this movie to the others?

Yes / No

4. Have you watched this clip before? 


\section{Part D:}

1. What is your overall rating of the clip (s)

( 1 is the worst while 10 is the best $)=$

2. Which plot/part is

a. the happiest?

b. the saddest?

c. the most memorable?

d. the most boring?

3. Will you recommend this movie to the others?

4. Have you watched this clip before?

\section{Part E:}

1. What is your overall rating of the clip (s)

( 1 is the worst while 10 is the best) $=$

2. Which plot/part is

a. the most exciting?

b. the happiest

c. the saddest?

d. the most memorable?

e. the most boring?

3. Will you recommend this movie to the others?

Yes / No

4. Have you watched this clip before?

Yes / No

Thank you for your participation, pls return this questionnaire to the researcher 